\title{
Dual-color chromogen In Situ hybridization (CISH) for the determination of Her2 status
}

\section{in breast cancer tissue sections}

\author{
Melanie Evelyne Herre \\ Faculty of Health, Medicine and Life Sciences \\ melanieherre@online.de
}

\section{Abstract}

Background: A factor associated with breast cancer development is the mutation of the Her2/neu gene located on chromosome 17. Chromogen in situ hybridization (CISH) in which the color is generated with an enzymatic reaction is a promising alternative detection method to fluorescence in situ hybridization (FISH) which is nowadays used in clinical practice. The resulting color is stable and therefore usable for permanent storage, the use of a normal brightfield microscope is possible and the morphology of the sample can be evaluated more easily than with a fluorescence microscope. For the visualization of Her2/neu it is however important to also detect chromosome 17 and use it as a reference chromosome. The aim of the study is: to select the best substrates considering color production, background staining, reactivity and localization. Secondly to develop a dualcolor procedure visualizing Her2/neu and chromosome 17 to examine the possibility of using $\mathrm{CISH}$ as a standardized method for the detection of Her2 positive tumors and finally to compare these results with FISH. Methods: Single target CISH with eight substrates for the enzyme horseradish peroxidase (PO) and six substrates for alkaline phosphatase (AP) was performed on cervical cancer cell lines with centromere $1\left(\mathrm{C}_{1}\right)$ and 1p36 as targets. The best substrates were subjectively selected with the use of a brightfield microscope. The procedure was expanded to a dual-color detection on cells and formalin-fixed, paraffin embedded (FFPE) breast cancer tissue sections with Her2/neu and centromere 17 (C17) as targets. In this procedure the best conjugate detection systems were worked out. Results: In the single target detection procedure the substrates DAB, Vina Green and Seramun Grün for PO and Ferangi Blue and Vulcan Fast Red for AP were found to give the best color reactions. The dual-color detection on tissue sections showed strong and good distinguishable color reactions when combining the substrates Vina Green for $\mathrm{C}_{17}$ and 
Vulcan Fast Red for Her2. Results of CISH and FISH were comparable when using immersion oil for the detection of the fluorescent signals. Discussion and Conclusion: Vina Green for $\mathrm{C} 17$ and Vulcan Fast Red for Her2/neu are the best substrates considering background staining, color distinguishability and localization and give comparable results to FISH. The study gives a further step in the direction of introducing $\mathrm{CISH}$ as a standardized method for the detection of Her2/neu amplification.

\section{Keywords}

Her2/neu, breast cancer, chromogen in situ hybridization (CISH), dual-color $\mathrm{CISH}$, fluorescence in situ hybridization (FISH), cytochemical detection systems

\section{Introduction}

Breast cancer is the second most common cancer in the world and the most frequent cancer among women. The somatic mutation leading to Her2/neu amplification is a common mutation in breast cancer. This mutation is observed in up to $30 \%$ of breast cancer cases and is associated with poor treatment and survival (1). Her2/neu also called human epidermal growth factor receptor 2 (ErbB2) is located on the long arm of chromosome 17 (2). ErbB2 induces mitogenic stimuli through the interaction with its ligands (3). Due to its amplification or overexpression it becomes an oncogene and stimulates enhanced cell growth, survival of the cells and proliferation resulting in the development of a tumor $(4,5)$.

In case of Her2/neu positivity special, personalized treatment options can be chosen. Trastuzumab, a human-monoclonal antibody has already shown to enhance life expectancy of women with Herz/neu positive tumors.

Since the Her2/neu amplification plays an important role in breast cancer, Her2 testing is done in every woman newly diagnosed with breast cancer (6). Testing has in the past mostly been done with immunohistochemistry (IHC) in which the overexpression of the Her2 protein in parallel to the presence of hormone receptors has been measured. Nowadays it is tested with FISH in which the amplification of the Her2 gene is visualized. IHC is less reliable and accurate than FISH since it is a more subjective detection which is furthermore dependent on fixation times and antibodies used. FISH is a more objective detection as the actual copy number has to be counted, it is however more expensive since there is the need for a fluorescence microscope, a certified pathologist and also the reagent costs are higher $(7,8)$. Alternative accurate, reliable and objective methods which are less expensive than FISH should therefore be tested. 
A technique often used in the laboratory to detect the presence or absence of specific gene sequences is "in situ hybridization" (ISH). It is useful in visualizing genetic variations that are appropriate for cancer detection, prognosis and selection of the best medication $(9,10)$.

In ISH the DNA which has to be tested is at first denatured. Subsequently a DNA probe consisting of the complementary sequence to the sequence that has to be detected is added. The added probe is either detected directly when labeled with a signal or indirectly when labeled with a hapten that can afterwards be detected immunohistochemically by a signal labeled antibody. Finally the DNA to be tested is hybridized with the probe and excess probe is washed away to avoid unspecific binding. To gain a signal two ISH methods are mainly used:

The first one is FISH. In this method the probe or the antibody becomes labeled with a fluorescent dye like fluorescein isothiocyanate (FITC) or tetramethylrhodamine (TRITC) that can afterwards be visualized under a fluorescence microscope. The FISH procedure can be performed rapidly. It has a high resolution and high sensitivity. Furthermore it has low endogenous background and more than one target can be visualized simultaneously by using different fluorochromes. But despite of its many advantages there are also some problems with FISH. It is difficult to assess tissue morphology and the signal will fade after a relatively short time what makes archiving and long term storage impossible. Another disadvantage is the autofluorescence in FFPE tissue sections.

The second method is $\mathrm{CISH}$. In this method an antibody becomes labeled with an enzyme like e.g. alkaline phosphatase (AP) or horseradish peroxidase (PO) that is able to convert a substrate and thereby produces a color reaction. Advantages of $\mathrm{CISH}$ are that the resulting color is stable and therefore usable for permanent storage. The use of a normal brightfield microscope is possible what is favorable for routine analysis and the morphology of the sample can be evaluated more easily than with a fluorescence microscope $(11,12)$.

Since cancer is however often associated with the presence of an abnormal number of chromosomes in a cell (aneuploidy) and Her2/neu is located on chromosome 17 it is essential to determine the number of chromosome 17 and use it as reference chromosome.

Currently most CISH is performed as a single target procedure in which two different tissue sections are evaluated. In the case of Her2/neu and chromosome 17 this means that 
two slides are needed to calculate the Her2/C17 ratio. This method however is cost and time consuming $(2,12)$. It is therefore crucial to design a dual-color procedure visualizing two gene targets on one microscopic slide to be able to use it in routine analysis. The dualcolor procedure however gives rise to some detection specific problems: It is essential to select substrates that are clearly distinguishable from each other and may not change their color or dissolve after some days. Another problem is that samples for clinical use mostly consist of paraffin-embedded tissue sections and not of cells. For tissue sections immunochemical conjugates may not be too big since they will not be able to diffuse into the sections anymore. Moreover the endogenous enzyme activity has to be blocked to avoid nonspecific color reactions.

\section{Aims of the study}

1. Selection of the best substrates for a single color $\mathrm{CISH}$ procedure when considering precipitate color, background staining, reactivity, sharpness and localization of the signals.

2. Development of a dual-color CISH procedure aiming for the detection of centromere 17 and Her2/neu on FFPE breast cancer tissue sections by using some clinical samples to examine the possibility of launching $\mathrm{CISH}$ as a standardized method for the detection of Her2 positive tumors.

3. Comparison of $\mathrm{CISH}$ with FISH

\section{Material and methods}

\section{Cell and tissue samples preparation}

The human cervical cancer cell lines CasKi, SiHa and HeLa and (FFPE) tissue sections of the cervix and the breast were utilized for the identification of the best substrates in the $\mathrm{CISH}$ procedure.

\section{DNA Probes}

DNA probes for $\mathrm{C}_{1}$ (1912), 1p36, HPV $16, \mathrm{C}_{17}$ and Her2 and probe mixes consisting of $\mathrm{C}_{1} / 1 \mathrm{p}_{3} 6$ and $\mathrm{C} 17 / \mathrm{Her} 2$ labeled with digoxigenin (dig) or biotin (bio) were used for the single- and dual-color detection on cells as well as on tissue sections. Furthermore one directly labeled probe (C17 FITC/Her2 TRITC) for the FISH procedure was applied.

\section{Detection}

To find the best detection systems different combinations consisting of antibody layers, polymers or an avidin detection system were tested. The final layer was either conjugated with the enzymes AP and PO or a fluorescent dye (FITC or TRITC). 


\section{Enzyme substrates}

Eight substrates for the enzyme PO (SeramunGrün ${ }^{\circledR}$ chip (green color; Seramun Diagnostica $\mathrm{GmbH}$ ), Vina Green ${ }^{\mathrm{Tm}} \mathrm{Chromogen}$ kit (green color; Biocare Medical), DAB chromogen/ Substrate kit (brown color; DAKO), Deep Space Black ${ }^{\text {TM }}$ Chromogen kit (black color; Biocare Medical), AEC (red/brown color; Pan Path), DAB chromogen/Substrate kit (brown color; Scy Tek), TrueBlue ${ }^{\mathrm{TM}}$ (blue color; Nutacon), Romulin AEC Chromogen kit (reddish-orange Biocare Medical)) and six substrates for the enzyme AP (Ferangi Blue ${ }^{T M}$ Chromogen kit 2 (blue color; Biocare Medical), Vulcan Fast Red Chromogen kit 2 (red color; Biocare Medical), NBT/BCIP (blue color; Pan Path), Warp Red ${ }^{\mathrm{TM}}$ Chromogen kit (red color; Biocare Medical), Alk-Phos Blue Kit (blue color; Scy Tek), New Fuchsin Kit (red color; Scy Tek)) were tested.

\section{Dual-color CISH procedure}

In the dual-color procedure antibodies for the bio and dig labeled probe mixes were simultaneously incubated. The following substrate combinations were tested: Ferangi Blue - DAB DAKO and v.v., Vulcan Fast Red - Vina Green and v.v., Vulcan Fast Red - Seramun Grün and v.v.

\section{Microscopic evaluation}

Colors were evaluated under a brightfield microscope (Zeiss, West Germany, Axioskop) or a Leica DM RBE fluorescence microscope.

\section{Results}

Substrate selection on cells

The single color detection was initially tested on CaSki cells with $\mathrm{C}_{1}$ and $1 \mathrm{p}_{3} 6$ as targets. Seramun Grün, Vina Green (Figure 1), DAB DAKO and Deep Space Black were found to be the best PO substrates when considering color production, sensitivity and background staining. The chosen substrates produced strong and clear dark green, green, brown and black colors. Ferangi Blue and Vulcan Fast Red (Figure 1) turned out to be the best AP substrates. The use of a two layer detection system consisting of a mono- or polyclonal antibody together with one of the polymers (Nichirei Biosciences INC) demonstrated to give strong, well detectable color spots with good localization.

After the selection of the best substrates the procedure was performed on HeLa cells with the goal targets $\mathrm{C}_{17}$ and Her2/neu. All $\mathrm{C}_{17}$ stainings were performed with PO substrates. In these reactions Seramun Grün showed to change its color to brown after some hours. Deep Space Black was found to have a bad reproducibility since not all nuclei 


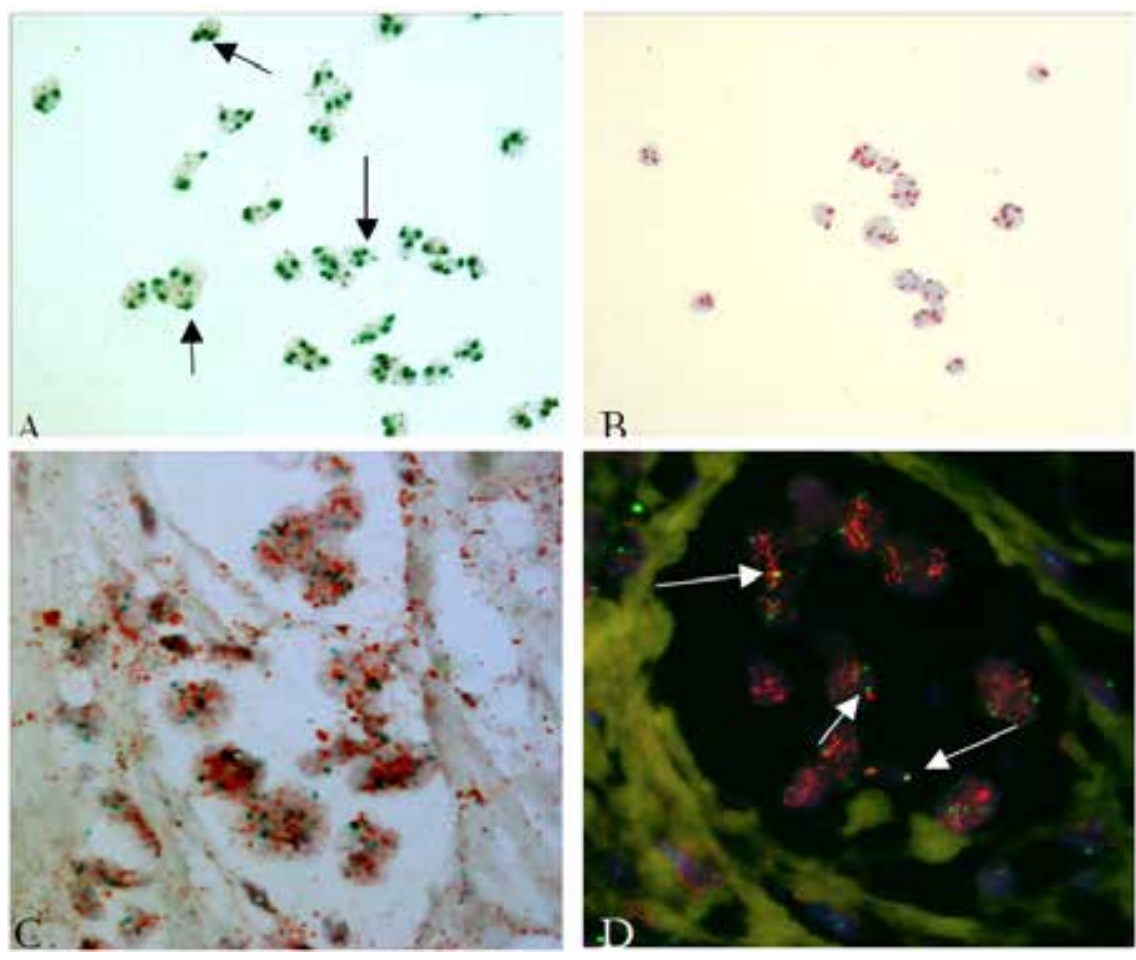

Figure 1. Brightfield microscopical detection of (A, B) single color CISH on CaSki cells and dual color CISH (C) and FISH (D) on FFPE breast cancer tissue sections. (A) Chromosome 1 (indicated by black arrows) detection with the peroxidase substrate Vina Green. (B) Chromosome 1 detection with the alkaline phosphatase substrate Vulcan Fast Red. Dual-color detection of chromosome 17 and Her2 with PO/Vina Green and AP/ Vulcan Fast Red respectively (C). Dual-color fluorescence detection of chromosome 17 (indicated by white arrows) and Her2 with FITC and TRITC respectively (D). C and D show a highly amplified Her2 gene and chromosome 17 polysomy.

DAB together with Ferangi Blue obtained a good reproducibility and localization without background signal and colors appeared to be clear and discernible. In the combination of Seramun Grün and Vulcan Fast Red, the slides appeared to have a strong background staining and the substrates were not distinguishable since Seramun Grün became brown which made the distinction from Vulcan Fast Red impossible. Vina Green together with Vulcan Fast Red showed easy distinguishable color signals with good localization and reactivity. The slides could however neither be embedded in Entellan nor in glycerol PBS since Vina Green dissolves in aqueous medium and Vulcan Fast Red is not stable in Entellan. The use of a dual layer detection system consisting of a mono- or polyclonal 
antibody together with one of the polymers was considered to give proper signal intensity with good localization and no background staining in all substrate combinations except from Seramun Grün together with Vulcan Fast Red which produced a high background staining on the glass slides.

\section{Single color CISH on FFPE-tissue sections}

Since Vina Green turned out to be the best substrate for the detection of $\mathrm{C}_{1}$, it was also tested for the visualization of $\mathrm{C} 17$. To enhance signal intensity, localization and decrease background staining, different detection systems were tested. It turned out that the avidin-PO system (avidin PO - bio GAA - avidin PO) gave a very strong, green signal with good reproducibility and localization and no background staining as shown in the $\mathrm{C}_{1}$ detection. Vulcan Fast red which gave red signals with good reactivity and high signal intensity was chosen to be the best substrate for Her2/neu. After a two-fold dilution of the probe, the background staining of Vulcan Fast Red became less but was still present.

FISH procedures were performed in addition to $\mathrm{CISH}$. The detection of $\mathrm{C}_{1}$ and $\mathrm{C}_{17}$ on tissue sections with an avidin-PO system (Vina Green) and an avidin FITC system showed comparable results. Both targets gave strong signals with good reproducibility in the $\mathrm{CISH}$ as well as in the FISH procedure. The detection of $\mathrm{C}_{17}$ showed a light background staining in both procedures. When using a one layer avidin PO and avidin FITC system for $\mathrm{C} 17$ the enzyme reaction was even more sensitive compared to FISH and the signal was stronger under the brightfield microscope. To get results with comparable resolution and brightness for FISH, immersion oil had to be used.

\section{Dual-color CISH on FFPE tissue sections}

For the dual-color $\mathrm{CISH}$ procedure on tissue sections the previously selected best substrates for $\mathrm{C}_{7} 7$ and Her2/neu, Vina Green and Vulcan Fast Red respectively, were tested. When at first stained with Vina Green the green color dissolved after several washing steps and the staining with Vulcan Fast Red. When Vulcan Fast Red was incubated first, both colors stayed stable (Figure 1).

For the comparison of fluorescence with the dual-color $\mathrm{CISH}$ detection of Her2 and $\mathrm{C}_{17}$ a probe directly labeled with FITC and TRITC was used (Figure 1). FISH as well as CISH showed background for Her2. The enzyme reaction however gave stronger signals although the concentration of the fluorescence probe had been twice as big as the concentration for the CISH probe. 


\section{Discussion/Conclusion}

For the substrate selection a model consisting of $\mathrm{C}_{1}$ and 1 p3 6 with known target size showed to be successful to select the most promising color combinations. Seramun Grün showed to be the best peroxidase (PO)/substrate but it later turned out that the dark green color was not stable but changed to brown and could therefore not be used in the dual-color staining with the red Vulcan Fast Red substrate due to difficult color separation. The influence of $\mathrm{pH}$ on the color was tested but no connection could be found. A solution for the stability problem should be investigated in future studies since Seramun Grün could be used as a substrate for the dual-color procedure due to its properties.

For the dual-color procedure with Vulcan Fast Red (VFR) and Vina Green (VG) several adaptations had to be made: VG is not stable in aqueous medium, it is therefore crucial to incubate VFR first to expose VG to as little washing steps as possible. Secondly VG is normally dehydrated in an ethanol series before being embedded. This is however not possible in the dual-color procedure since VFR would dissolve. Moreover embedding of VG in PBS glycerol is not possible because it dissolves in aqueous solutions. Since VFR showed to dissolve in Entellan none of these embedding media can be used. A possible solution has been shown to be the embedding medium 'PanMount' (PanPath). To obtain stable colors it showed to be essential to embed the tissue section at first in this medium, let them air-dry and subsequently embed them in Entellan.

Our final detection system for Her2 still showed background staining despite of the reduced probe concentration. This is no problem if Her2 is highly amplified or present as amplicon because the calculation of the Her2/ $\mathrm{C}_{17}$ ratio is not necessary as it can be seen without counting the exact gene number. In case of a lower ratio calculation of the Her2/ $\mathrm{C}_{17}$ ratio is however important and background staining has to be removed. A reason for the high background noise could be the length of the probe. Large DNA probes are desirable because they give greater hybridization efficiency and more intense signals. Our probes showed to have a DNA length of 150-400 nucleotides. A problem of these probes is however that they contain repetitive DNA sequences that can bind to common repetitive DNA sequences in the cells or tissue sections of the patient. These unspecific probe bindings cause background staining and make the calculation of the Her2/ $\mathrm{C}_{17}$ ratio impossible. A possible solution is the use of a technique called chromosomal in situ suppression (CISS) hybridization (13). 
In summary it can be stated that the use of dual-color CISH as a standardized method for the detection of Her2 positive breast tumors is conceivable. This study however only gives a starting point for further research in which different problems like e.g. the background staining have to be improved. Furthermore the findings have to be confirmed with larger studies using bigger amounts of samples.

\section{Role of the student}

Melanie Herre was an undergraduate student biomedical sciences, with a specialization in molecular life sciences, working under the supervision of Dr. Anton H.N. Hopman when the research in this report was performed. The collection of data, the processing of the results as well as the formulation of the conclusions and the writing were done by the student.

\section{References}

1. John HMB, Ch B. Trastuzumab Use in Breast Cancer: Clinical Issues. 2002;9(6):499-507.

2. Nitta $H$, Hauss-Wegrzyniak B, Lehrkamp $M$, Murillo $A E$, Gaire $F$, Farrell $M$, et al. Development of automated brightfield double in situ hybridization (BDISH) application for HER2 gene and chromosome 17 centromere (CEN 17) for breast carcinomas and an assay performance comparison to manual dual color HER2 fluorescence in situ hybridization (FISH). Diagnostic pathology. 2008;3:41. PubMed PMID: 18945356. Pubmed Central PMCID: 2577627.

3. FantI WJ, Johnson DE, Williams LT. Signaling by receptor tyrosine kinases. Annu Rev Biochem. 1993;62:453-81.

4. Chang J-LMD, Tsao Y-PMDPD, Liu D-WMD, Han C-PMDSD, Lee W-HMDSD, Chen S-LPD. The Expression of Type I Growth Factor Receptors in the Squamous Neoplastic Changes of Uterine Cervix. Gynecologic Oncology. 1998;73:62-71.

5. Piccart-Gebhart MJMDPD, Procter MMS, Leyland-Jones BMDPD, Goldhirsch AMD, Untch MMD, Smith IMD, et al. Trastuzumab after Adjuvant Chemotherapy in HER2Positive Breast Cancer. N Engl J Med. 2005;353(16):1659-72.

6. HER2 Testing for Breast Cancer: ASCO; 2015. Available from: http://www.cancer.net/ research-and-advocacy/asco-care-and-treatment-recommendations-patients/her2testing-breast-cancer.

7. Dendukuri NP, Brophy J. Testing for Her2 positive breast cancer: a cost-effectiveness analysis. 2006 (23):1-59.

8. Carlson B. Her2 tests: How do we choose? Biotechnology Healthcare. 2008:23-7. 
9. Garcia-Caballero T, Grabau D, Green AR, Gregory J, Schad A, Kohlwes E, et al. Determination of HER2 amplification in primary breast cancer using dual-colour chromogenic in situ hybridization is comparable to fluorescence in situ hybridization: a European multicentre study involving 168 specimens. Histopathology. 2010 Mar;56(4):472-80. PubMed PMID: 20459554. Pubmed Central PMCID: 2855864.

10. Chen AY, Chen A. Fluorescence in situ hybridization. The Journal of investigative dermatology. 2013 May;133(5):e8. PubMed PMID: 23594542.

11. Hopman AHN, Claessen S, Speel EJM. Multi color brightfield in situ hybridisation on tissue sections. Histochem Cell Biol. 1997;108:291-8.

12. Kato N, Itoh H, Serizawa A, Hatanaka Y, Umemura S, Osamura RY. Evaluation of HER2 gene amplification in invasive breast cancer using a dual-color chromogenic in situ hybridization (dual CISH). Pathology international. 2010 Jul;60(7):510-5. PubMed PMID: 20594272.

13. Diagnostics R. COT Human DNA Switzerland: ROCHE; 2015. Available from: https:// lifescience.roche.com/shop/products/cot-human-dna. 